\title{
Experimental and Numerical Quantification of Radial Flow in the Root Region of a HAWT
}

\author{
Buşra Akay, ${ }^{*}$ Carlos S. Ferreira, ${ }^{\dagger}$ Gerard van Bussel ${ }^{\ddagger}$ \\ DUWIND, Delft University of Technology, Delft, 2619HS, The Netherlands \\ Ivan Herraez ${ }^{\S}$ \\ ForWind, Carl von Ossietzky University Oldenburg, Oldenburg, 26129, Germany
}

\begin{abstract}
This paper explores the evolution of radial flow in a Horizontal Axis Wind Turbine (HAWT) blade root region. The radial flow is analyzed in the potential flow and viscous flow regions. An experiment carried out by means of stereo Particle Image Velocimetry to measure the velocity field produced by a HAWT blade. While the radial flow in the potential flow region was obtained from the measurements, the radial flow in the boundary layer was derived from CFD. By the direct observations obtained from the experiment, an insight is gained about the nature of the radial flow in the suction side of the blade as well as in the near wake. An outboard radial flow motion is observed in the root region. This tendency of the flow changes dramatically when it reaches the maximum chord position of the blade, where the radial flow moves inboard. The trace of the viscous region due to merging of the boundary layers and trailing vorticity are observed clearly in the radial velocity and vorticity distributions at $135^{\circ}$ azimuth angle of the blade. In the viscous flow region the radial flow is more pronounced than in the potential flow region. The performed CFD simulations are able to predict the vortex formation in the maximum chord region and its interaction with the nacelle.
\end{abstract}

\section{Nomenclature}

$\begin{array}{ll}\text { HAWT } & \text { Horizontal Axis Wind Turbine } \\ P I V & \text { Particle Image Velocimetry } \\ 3 C & \text { Three components } \\ \text { OJF } & \text { Open Jet Facility } \\ F O V & \text { Field of view } \\ s & \text { Field of view around the blade } \\ w & \text { Field of view in the wake } \\ \omega & \text { Vorticity } \\ U_{\infty} & \text { Inflow velocity } \\ V_{r} & \text { Radial velocity component } \\ V_{\theta} & \text { Circumferential velocity component } \\ \Omega & \text { Azimuth angle } \\ r & \text { Local radial position } \\ R & \text { Rotor radius } \\ x & \text { Position in the wake } \\ c & \text { Chord }\end{array}$

*PhD researcher, Wind Energy Group, Kluyverweg 1 2619HS Delft, The Netherlands.

$\dagger$ Assistant Professor, Wind Energy Group, Kluyverweg 1 2619HS Delft, The Netherlands.

¥Professor, Wind Energy Group, Kluyverweg 1 2619HS Delft, The Netherlands.

$\S$ PhD researcher, Research Group TWIST, Ammerländer Heerstr. 13626129 Oldenburg, Germany. 


\section{Introduction}

In this piece of research work, we describe the flow field around a Horizontal Axis Wind Turbine (HAWT) blade in two parts; the viscous flow field (the boundary layer), and the potential flow field (inviscid near wake). The authors' previous experimental and numerical studies showed a considerable radial flow motion in the potential flow field at the tip and at the root regions of the blade where the directions corresponding to the orientations of the tip and root vortices $\left({ }^{1,2}\right)$. This previous observations led us work on the radial flow evolution in the root region of the blade in detail.

\section{A. Background}

It must be noted that much of the interest in the literature is on the role of radial flow in stall delay mechanism. In a rotating system, radial flow has different characteristics than that in a stationary case. In the case of a wind turbine blade root region, radial flow causes Coriolis force which has tendency to push the flow in the chordwise direction towards the trailing edge. This mechanism is called stall delay or rotational augmentation. It postpones the point of separation and creates a significant increase in lift coefficient which was firstly observed in the famous experiment of Himmelskamp. ${ }^{3}$ The early investigations on rotational effects did not show a significant effect on the boundary layer development in the attach flow region. ${ }^{4}$ Later experiments supported the hypothesis that the rotational effects are more pronounced in the separated flow region than in the attached flow region. ${ }^{5}$ Moreover, some researches indicated the importance of blade geometry coupled with blade rotation for stall delay. ${ }^{6}$ This may also be supported by the other observations $^{7,8,9}$ showed an apparent stall delay phenomenon at the inner part of the blade where high angles of attack occur.

In the potential flow field, the radial flow are less dominant than that in the boundary layer. However, it still carries the flow characteristics into the inviscid region. The outboard motion of the radial flow may be noted in the velocity measurements of Ebert and Wood. ${ }^{10}$ Medici et. al ${ }^{11}$ also mentioned the expansion in the wake by showing an outboard motion. However, in the core region the radial velocity was in the inboard direction.

The aim of the present work is to link results that are based on direct observations of radial flow developed in the potential flow region and based on computations. Finally, this study contributes:

1. to the quantification of the radial flow enhanced in the potential flow field,

2. to the investigation of the driving mechanisms of radial flow in the potential flow field,

3. to the knowledge of three dimensionality of the flow field in the root region of a HAWT blade.

The following sections contain the description of the rotor model, the stereo Particle Image Velocimetry (PIV) experimental set-up, the methodology of the numerical analysis, the results, the discussions and the conclusions.

\section{Methodology}

The investigation was performed by means of stereo PIV and by means of Computational Fluid Dynamics (CFD) computations which can provide detailed information in the boundary layer. PIV was already proven that it is a powerful and non-intrusive method as a measurement technique in the wind tunnel tests. It gives quantitative, instantaneous velocity data on the field of interest. Moreover, three components (3C) of velocity can be measured by stereo PIV. The only disadvantage of the method applying such a problem is not having boundary layer information due to reflections on the surface of the blade. However, there are also methods improved to solve this deficiency of PIV. In this study, we use CFD to get the boundary layer information.

\section{A. Experimental Procedure}

\section{Wind Tunnel, HAWT Model, Measurement Conditions}

The experimental work was performed at the Open Jet Facility (OJF) at Delft University of Technology by means of stereo PIV to measure the flow field around a HAWT blade. OJF is a closed circuit wind tunnel 
which has a test section with $6 \mathrm{~m}$ width, $6.5 \mathrm{~m}$ height and $11 \mathrm{~m}$ length in the inflow direction. It operates up to $30 \mathrm{~m} / \mathrm{s}$. The rotor model used in the experiment represents a horizontal axis, 2 bladed wind turbine model with a $1 \mathrm{~m}$ radius. The chord and twist distribution of the blade is represented in Figure 1 . The blade model has a $0.0643 \mathrm{~m}$ chord length and $0^{\circ}$ twist at the tip and a $0.2027 \mathrm{~m}$ maximum chord length and a $16^{\circ}$ twist at the root. The Reynolds number around the maximum chord is 220000 . The operating conditions of the model during measurements are tabulated in Table 1.

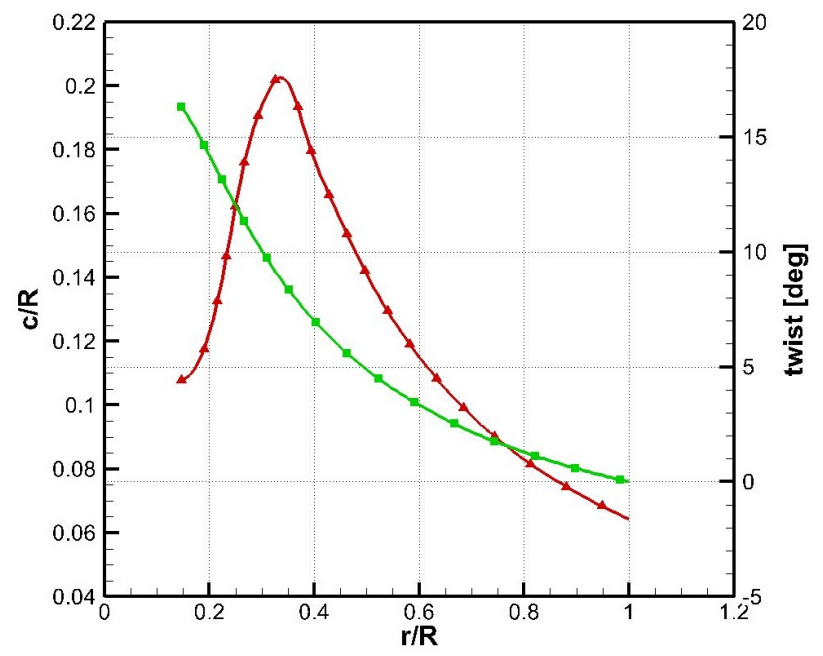

Figure 1. Chord and twist distribution of the model rotor blade.

Table 1. Measurement conditions.

\begin{tabular}{ccccc}
$\lambda$ & $\omega[\mathrm{RPM}]$ & $U_{\infty}[\mathrm{m} / \mathrm{s}]$ & $C_{T}$ & $C_{M}$ \\
\hline 7 & 400 & 6 & 0.62 & 0.069
\end{tabular}

\section{PIV measurements}

The Stereo PIV technique was applied in order to obtain 3C of velocity on the two dimensional planar fields of view (FOV) around the blade and in the near wake. The flow is seeded with fog particles generated by SAFEX Twin Fog generator using SAFEX-Inside-Nebelfluid which is a mixture of diethylene glycol and water. The size of the seeding particles is around 1 micron median diameter. Seeding was actualized in the downstream of the measurement area. Hence, a homogenous mixture of the seeding in the flow was obtained in the test room. The FOV was illuminated by double cavity pulsed Nd:YAG laser. The laser sheet had 3mm thickness. Two 16 mega pixel cameras with $180 \mathrm{~mm}$ lenses were used with Davis 7.2 software for acquisition of the images. The aperture value of the lenses was set to $\sharp \sharp 5.6$ during the measurements. The cameras and the laser were fixed on a computerized traverse system so that they moved together. Therefore, it was not necessary to do calibrations at each FOV position. The synchronization between the laser pulses and the position of the rotating blade was achieved for the designed experiment with a triggering system inserted in the hub. The measurement set-up in OJF is presented in Figure 2.

The azimuth angle of the blade is $0^{\circ}$ when the blade is up position (see Figure 3 -a). The measurements were performed on the plane at $90^{\circ}$ position when the blade was at different azimuth position by assuming axisymetric flow field around the rotor. Hence, the measurement plane was in the stationary frame of reference. The specified FOVs are represented in Figure 3-b. The measurement planes abbreviated as $s$ represents the planes around the blade, $w$ represents the planes in the near wake. Measurements were performed from $45^{\circ}$ to $180^{\circ}$ of the azimuth angle at every $5^{\circ} .200$ and 100 phase-locked image couples were respectively recorded at each measurement position of the $s$ and $w$ regions with a $100 \mu$ s separation time. The PIV apparatus and measurement conditions are summarized in Table 2. 


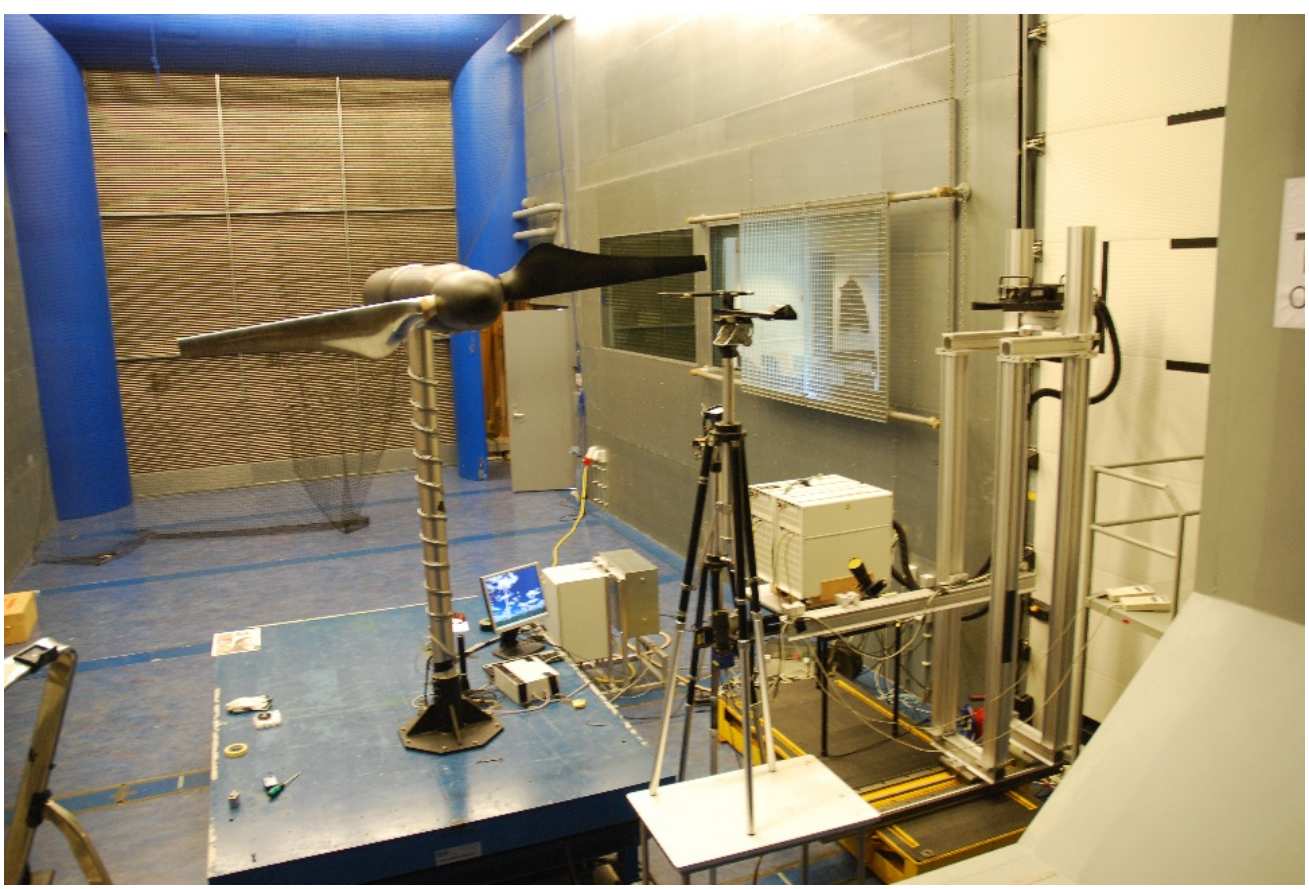

Figure 2. Measurement set-up in OJF.

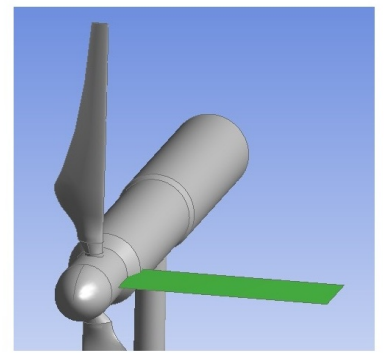

(a)

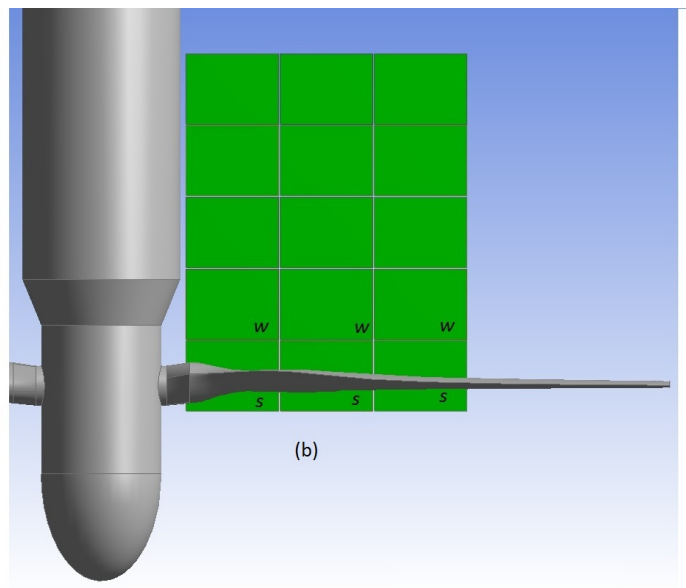

Figure 3. (a) Blade is at $0^{\circ}$ azimuth angle and the measurement plane is at $90^{\circ}$. (b) Measured fields of view around the blade, $s$ and in the wake, $w$.

\section{B. Numerical Approach}

The simulation model includes the rotor blades, hub and nacelle of the wind turbine model. The tower is believed to play a negligible effect on the rotor flow, so it was not modeled. The blockage ratio of the wind tunnel is small enough for avoiding tunnel effects. Therefore, including its walls in the model would be superfluous. A RANS approach based on the "frozen rotor" concept was chosen for the computations. The "frozen rotor" method allows to obtain a steady-state solution for a given rotor-stator configuration. Since in this case the tower and the wind tunnel walls are not part of the model, the obtained results are not related to any specific azimuth angle of the rotor. The simulations were performed with the free source finite volume CFD package OpenFoam. The multiple reference frame, incompressible an isotherm solver MRFSimpleFoam was employed with a non-inertial reference frame for the rotor. The rotation of the blades 
Table 2. PIV apparatus and measurement parameters.

\begin{tabular}{|c|c|c|c|}
\hline $\begin{array}{l}\text { Seeding } \\
\text { Laser } \\
\text { Camera } \\
\text { Lens }\end{array}$ & $\begin{array}{l}\text { SAFEX-Inside-Nebelfluid } \\
\text { Nd-Yag } \\
\text { Lavision Image Pro LX } \\
\text { Nikon }\end{array}$ & $\begin{array}{l}\text { Particle size } \\
\text { Laser sheet thickness } \\
\text { Camera resolution } \\
\text { focal length } 180 \mathrm{~mm}\end{array}$ & $\begin{array}{l}1 \mathrm{micron} \text { median diameter } \\
3 \mathrm{~mm} \\
16 \mathrm{Mega} \text { pixel } \\
\mathrm{f} \sharp 5.6\end{array}$ \\
\hline & $\begin{array}{l}\text { Acquisition software } \\
\text { Separation time } \\
\text { Number of image couples } \\
\text { FOV } \\
\text { Post-processing software } \\
\text { Window size [pix] } \\
\text { Final vector spacing }\end{array}$ & $\begin{array}{l}\text { DAVIS } 7.2 \\
100 \mu \mathrm{s} \\
200 \text { at } s, 100 \text { at } w \\
251 \mathrm{~mm} \times 168 \mathrm{~mm} \\
\text { Davis } 7.4 \\
\text { Decreasing from } 64 \times \\
0.605 \mathrm{~mm}\end{array}$ & 64 to $16 \times 16$ \\
\hline
\end{tabular}

is accounted for by adding the Coriolis and centripetal forces to the momentum equations. The major advantage of this method is that no moving meshes are required. A comparatively fast computation can therefore be achieved. The SIMPLE solution algorithm is utilized for the pressure-velocity coupling. The mesh was generated with the tool snappyHexMesh, which is included in OpenFoam. About 9 million cells of type hexaedra and split-hexahedra are required in total for resolving finely enough the rotor and near wake area. Since the focus of this work is the study of radial flows on the blades, the mesh coarsens rapidly after about 1.5 meters downstream of the rotor. The boundary layer on the blades is resolved with 10 cell layers. In the case of the hub and the nacelle, 5 cell layers are used. The expansion ratio of those cells in the normal direction to the walls is 1.25 . In order to guarantee a good functioning of the wall functions, the non dimensional wall distance value $y+$ ranges from 30 to 100 . The considered wall functions correspond to the utilized eddy viscosity model, which is the k-omega SST. The selected discretization schemes are of second order. No periodic boundary conditions are considered because the same mesh should be used in future for transient simulations. The simulations were run with 380 processors in a computer cluster of the University of Oldenburg.

\section{Results}

\section{A. Radial velocity distribution}

Figure 4 represents a spanwise cut of the blade \& wake through $90^{\circ}$ position when the blade is also at $90^{\circ}$ and at $135^{\circ}$ azimuth positions respectively. In these figures, non-dimensionalized phased averaged radial velocity distributions are presented around the blade and in the near wake of the blade at each azimuth position. To be able to plot this whole velocity field, the separate measurements performed at $s$ and $w$ planes combined without any interpolation at the connection edges. The connections are represented by dashed lines on the contour plots. Although some discontinuities may be noted at the FOV connections, this does not affect the information extracted from the velocity field. Computational results are represented in two planes, $90^{\circ}$ and $135^{\circ}$ in Figure 5. The axis of the plots are non-dimensionalized by radius of the rotor. To clarify the coordinate system used in the figures, it is worthwhile to address the directions of the flow. The axial/freestream flow is in the direction of downstream which is in $+x / R$, the positive radial flow is from root to tip and finally the positive tangential flow is in the out of plane (FOV) direction.

A close-up view to the boundary layer flow is shown in Figure 6. The dashed line on the contour plots shows the mask on the PIV image. The same line is also inserted onto CFD result to indicate the region where we do not get any information from PIV results.

\section{B. Coherent structures}

The radial velocity distribution is already a kind of footmark of the vorticity structures in the near wake of the blade. Moreover, there are several criteria to detect the coherent structures from the velocity data ${ }^{12,13,} .{ }^{14}$ The applied method to extract the flow structures is mainly depend on the type of the velocity field. For 

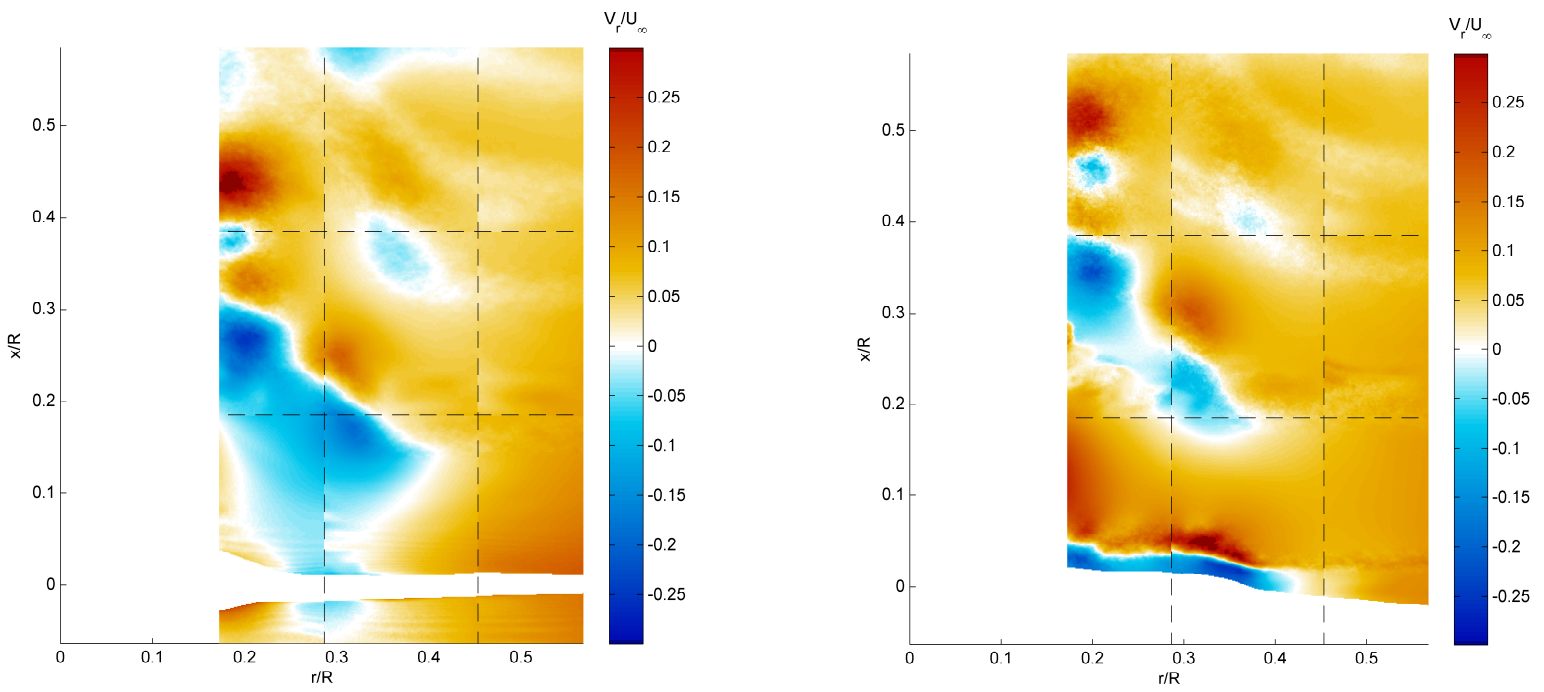

Figure 4. Radial velocity contour non-dimensionalized by $U_{\infty}$ (PIV), blade position at $\Omega=90^{\circ}$ (left) and $\Omega=135^{\circ}$ (right).


Figure 5. Radial velocity contour non-dimensionalized by $U_{\infty}$ (CFD), plane at $\Omega=90^{\circ}$ (left) and $\Omega=135^{\circ}$ (right).

example, in wall-bounded flows, the main problem is the discremination of the vorticity due to shear flow from the vorticity due to vortical flow. We performed a short study to detect the coherent structures in the near wake of the blade. We apply two methods for that purpose; vorticity and $\lambda_{2}$ calculations on the averaged velocity data. Jeong and Hussain ${ }^{12}$ showed that $\lambda_{2}$ has a negative value in the core of the vortex. The comparison of these two methods shows that the flow in the blade wake is unbounded. Figure 7 presents the vorticity distribution obtained by PIV and CFD when the blade is at $90^{\circ}$ azimuth position. Vorticity distribution when the blade reaches $135^{\circ}$ azimuth position is presented in Figure 8 .

Vorticity, $\omega_{z}$, is derived from the phased averaged velocity distribution using;

$$
\omega=\frac{\partial v}{\partial x}-\frac{\partial u}{\partial y}
$$

where $\mathrm{u}$ and $\mathrm{v}$ are the velocity components in the radial and axial directions respectively.

$\lambda_{2}$ criterion states that in the core of a vortex $\lambda_{2}<0$. 

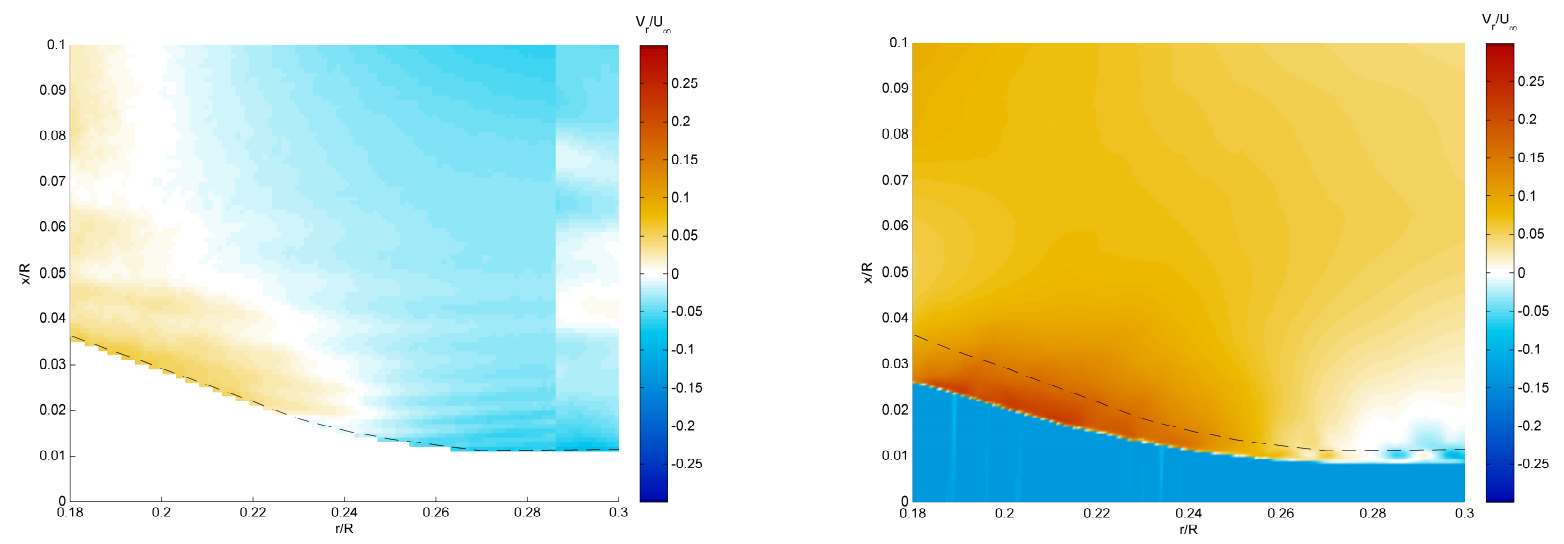

Figure 6. Radial velocity contour non-dimensionalized by $U_{\infty}$, blade position at $\Omega=90^{\circ}$, PIV (left) and CFD (right).

$$
\omega=\left(\frac{\partial u}{\partial x}\right)^{2}+\left(\frac{\partial u}{\partial y} * \frac{\partial v}{\partial x}\right)<0
$$
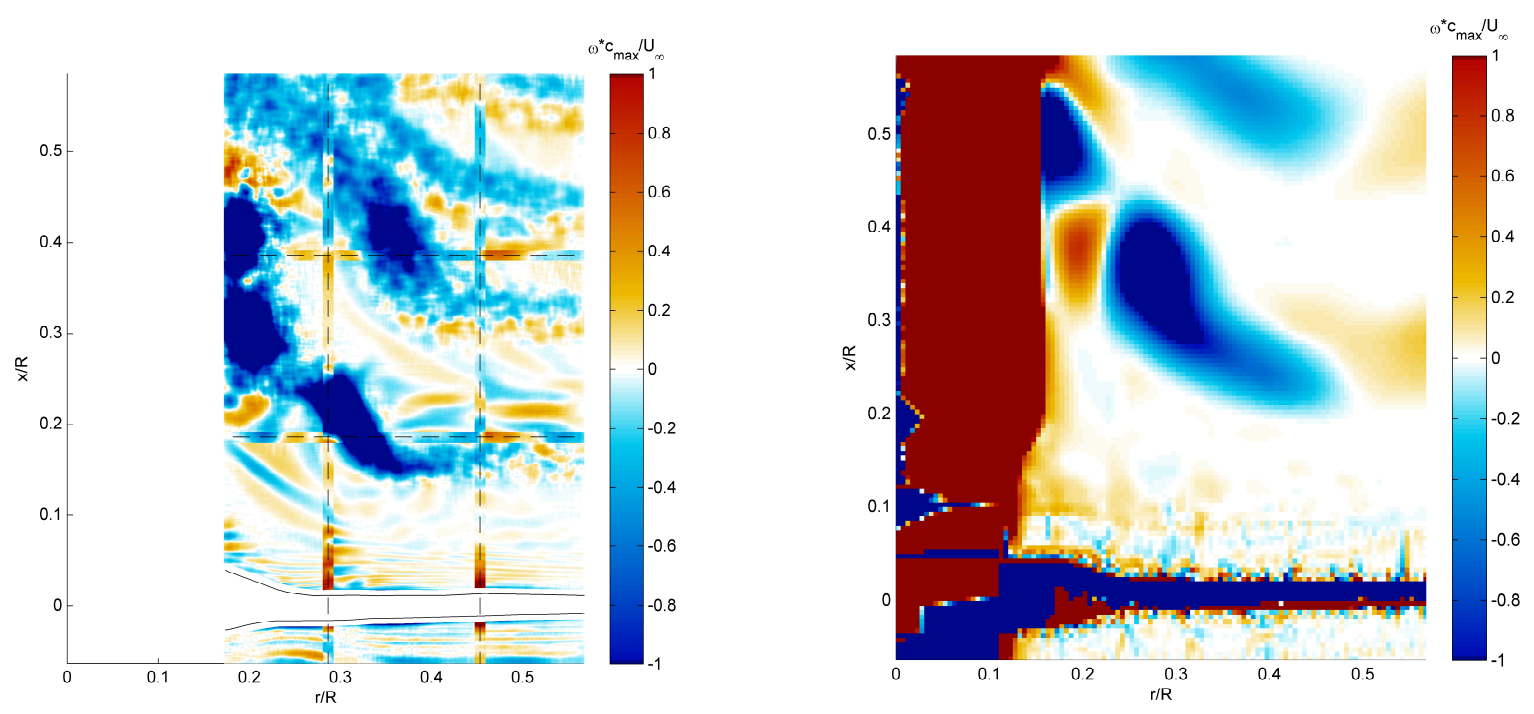

Figure 7. Vorticity distribution, PIV (left) and CFD (right), blade position at $\Omega=90^{\circ}$.

\section{Discussions}

It is possible to observe the evolution of the blade wake by analyzing the results at the different azimuth positions of the blade. Here, we only present the results for the $90^{\circ}$ and $135^{\circ}$ blade azimuth angles. A weak outboard motion in the radial flow is observed in the left plot of Figure 4 up to $r / R=0.25$. This motion extends in the wake until $\mathrm{x} / \mathrm{R}=0.19$. However, in between $\mathrm{r} / \mathrm{R}=0.25$ and $\mathrm{r} / \mathrm{R}=0.35$ where the maximum chord located, an inboard motion of the radial flow is noted. It is observed that this inboard motion is connected with the hub vortex in the near wake. Inboard behavior of the radial flow was also observed in Medici. ${ }^{11}$ However, they did not mention the outboard motion at very close region to the nacelle. After around $\mathrm{r} / \mathrm{R}=0.35$, the flow moves in the outboard direction which supports the fact of wake expansion just behind the rotor plane. The changes in the sign of the radial flow reflect the nature of the flow on the suction side of the blade as well as in the near wake. It is observed that the large inboard flow region at $90^{\circ}$ azimuth 

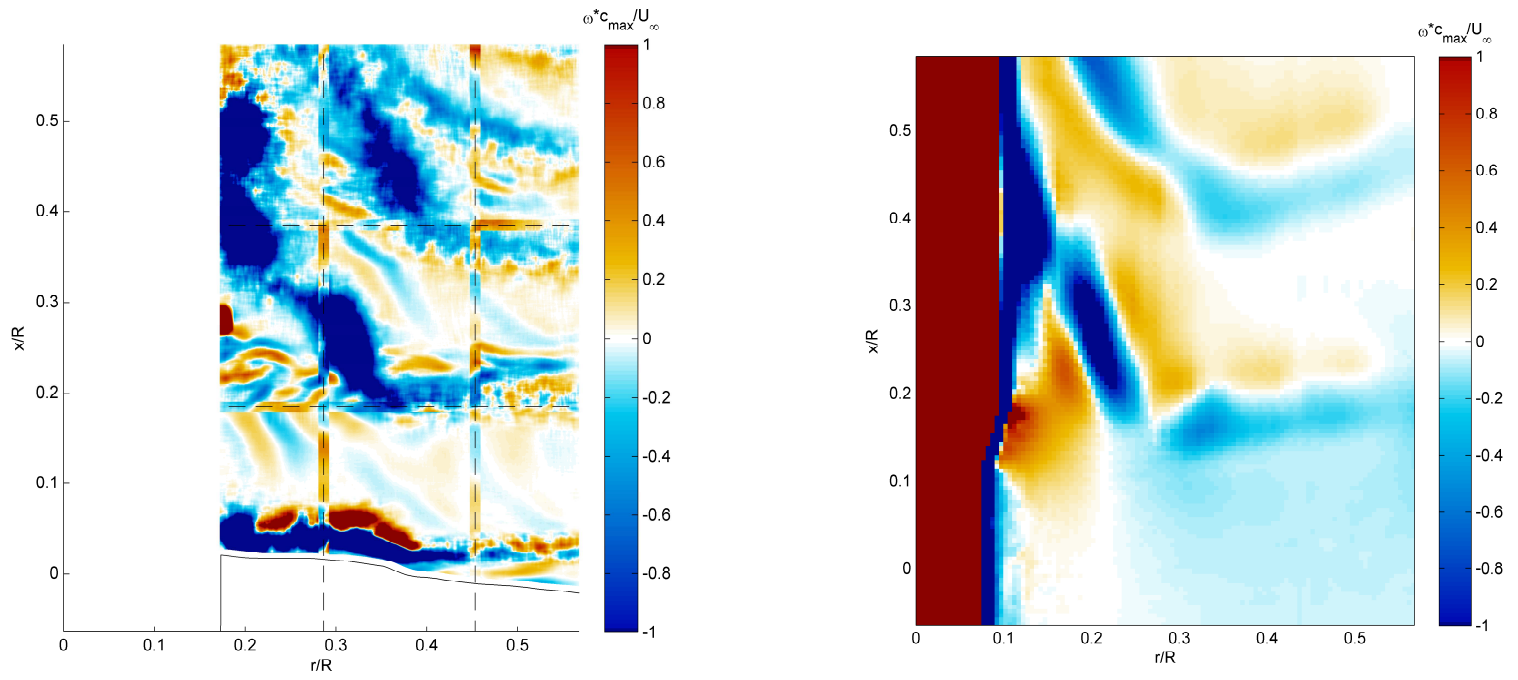

Figure 8. Vorticity distribution PIV (left), and CFD (right) blade position at $\Omega=135^{\circ}$. Data filtering is applied on PIV data for the visual aid.

position detaches from the blade at $135^{\circ}$ azimuth position (Figure 4-right).

The trace of the viscous flow region is clearly observed in Figure 4-right which is due to the merging of the boundary layers of suction side and the pressure side. When we look at the vorticity distribution at this position of the blade (see Figure 8) one may observe the trailing vorticity and two dominant vortex structures at the hub and at the maximum chord position. (These two vorticities are also observed in $\lambda_{2}$ distribution which is not shown here.) The position of the trailing vorticity observed in this figure is agreed very well with the position of the viscous wake of the blade. By knowing the rotational velocity of the rotor model, one may easily calculate the blade passage displacement onto $90^{\circ}$ measurement plane in the time interval from $90^{\circ}$ azimuth position to $135^{\circ}$ blade azimuth position. The axial velocity distribution shows around $3 \mathrm{~m} / \mathrm{s}$ in the blade wake. Therefore, the blade passage displacement is calculated $0.056 \mathrm{~m}$ in the wake in $45^{\circ}$ azimuth displacement. This simple calculation is confirmed by the experimental results. One may clearly observe this position in the blade wake on the velocity distributions as well as in the vorticity distributions. The blade passage (merging boundary layers) at $135^{\circ}$ azimuth angle creates negative and positive radial velocity in the blade wake, which induces a negative velocity distribution in the azimuth direction (see Figure 9). This velocity distribution shows that the wake of the rotor rotates in the opposite direction of the blade rotation, which is expected. The previous blade passages may also be observed with zero velocity distributions in the wake. In this figure it is also noted that at the $\mathrm{r} / \mathrm{R}=0.3$ location, a vortex releases from the maximum chord position which moves in radial direction.

The measurements show that the flow around the HAWT blade is highly non-stationary and partly governed by secondary flows. This makes certainly challenging to get a detailed flow description by means of steady state simulations, since this kind of simulations are actually better suited for stationary flows. The prediction capabilities of the numerical model are however reasonable if the mentioned limitations are taken into account. One of the main advantages of the simulations with respect to the measurements is that they allow to get a closer view in the boundary layer (see Figure 6). The performed simulations are able to predict the vortex formation in the maximum chord region and its interaction with the nacelle.

It is clearly noted that CFD simulation can not capture the whole area of the radial flow inboard motion which is observed in Figure 4-left. Also, the experimental results shows a much lower outboard radial motion compared to CFD simulation results. This observation was also mentioned in the comparison of PIV and panel model simulation by Micallef et. $\mathrm{al}^{1}$ These discrepancies between CFD and PIV results could be due to comparing steady-state simulations with experiments. It is certainly necessary to perform transient simulations for better understanding. 




Figure 9. Circumferential velocity distribution (PIV), blade position at $\Omega=135^{\circ}$.

\section{Conclusions}

An experimental campaign carried out by means of stereo PIV to investigate the evolution of radial flow produced by a HAWT blade. While the radial flow in the potential flow region was obtained from the measurements, the radial flow in the boundary layer was derived from CFD. The experimental and numerical results contribute to the understanding of the radial flow around the blade and in the near wake. The following points are highlighted in terms of velocity and vorticity distributions;

1. Outboard motion of radial flow at the root region is observed.

2. Two sets of vorticities are observed releasing from the root and the maximum chord position.

3. The trace of the viscous flow region generated by the boundary layers of suction side and the pressure side is noted.

4. The trailing vorticity is observed in the potential flow region which is coincide with the position of the viscous wake of the blade.

5. At the blade passage, a negative tangential velocity occurs due to positive and negative radial flow.

6. The experimental results shows a much lower outboard radial motion compared to CFD simulation results.

7. The performed CFD simulations are able to predict the vortex formation in the maximum chord region and its interaction with the nacelle.

\section{Acknowledgments}

This research project is supported by Vestas. 


\section{References}

${ }^{1}$ D. Micallef, B. Akay, T. Sant, C. Ferreira, and G. van Bussel, "Experimental and numerical study of radial flow and its contribution to wake development of a HAWT," in European Wind Energy Conference and Exhibition-EWEC, Brussels, 2011.

${ }^{2}$ B. Akay, D. Micallef, C. Ferreira, and G. van Bussel, "Stereo PIV experiments on Horizontal Axis Wind Turbine Rotor," in 46th Applied Aerodynamics Symposium, Orleans, France, 2011.

${ }^{3}$ H. Himmelskamp, "Profile investigations on a rotating airscrew," tech. rep., MAP Volkenrode, Reports and Translation. No:832, 1947.

${ }^{4} \mathrm{~J}$. Sørensen, "Prediction of three-dimensional stall on wind turbine blade using three-level, viscous-inviscid interaction model," European Wind Energy Association Conference and Exhibition, pp. 429-435, 1986.

${ }^{5}$ S. Schreck, N. Sørensen, and M. Robinson, "Aerodynamic structures and processes in rotationally augmented flow fields," Wind Energy, vol. 10, pp. 159-178, 2007.

${ }^{6} \mathrm{~S}$. Schreck and M. Robinson, "Horizontal Axis Wind Turbine blade aerodynamics in experiments and modeling," IEEE Transactions on Energy Conversion, vol. 22, pp. 61-70, 2007.

${ }^{7}$ D. Hu, O. Hua, and Z. Du, "A study on stall-delay for horizontal axis wind turbine," Renewable Energy, vol. 31, pp. 821-836, 2006.

${ }^{8}$ Z. Du and M. Selig, "The effect of rotation on the boundary layer of a wind turbine blade," Renewable Energy, vol. 20, pp. $167-181,2000$.

${ }^{9}$ H. Dumitrescu and V. Cardos, "Rotational effects on the boundary-layer flow in wind turbines," tech. rep., AIAA Journal-Technical Notes, 2003.

${ }^{10} \mathrm{P}$. Ebert and D. Wood, "The near wake of a model horizontal-axis wind turbine-I: Experimental arrangements and initial results," Renewable Energy, vol. 12, pp. 225-243, 1997.

${ }^{11}$ D. Medici and P. Alfredsson, "Measurements on a wind turbine wake: $3 \mathrm{~d}$ effects and bluff body vortex shedding," Wind Energy, vol. 9, pp. 219-236, 2006.

12 J. Jeong and F. Hussain, "On the identification of a vortex," J. Fluid Mech., vol. 285, pp. 69-94, 1995.

${ }^{13} \mathrm{P}$. Chakraborty, S. Balachandar, and R. Adrian, "On the relationships between local vortex identification schemes," J. Fluid Mech., vol. 535, pp. 189-214, 2005.

${ }^{14} \mathrm{H}$. Vollmers, "Detection of vortices and quantitative evaluation of their main parameters from experiemntal velocity data," Measurement Science and Technology, vol. 12, pp. 1199-1207, 2001. 